\title{
If only Poiseuille had had a computer
}

\author{
William M. DeCampli, MD, PhD
}

See related article on pages 689-96.

In this issue of the Journal of Thoracic and Cardiovascular Surgery, Schiavazzi and coworkers ${ }^{1}$ present their latest report using multiscale modeling (MSM) with computational fluid dynamics (CFD) to study congenital heart disease. Their work arises from a group called Modeling of Congenital Hearts Alliance (MOCHA), an alliance that has existed for more than a decade and serves as a prototype for productive collaboration between medicine and engineering.

In the current report of Schiavazzi and coworkers, ${ }^{1}$ they describe their studies of the effects of left pulmonary artery (LPA) stenosis on the superior cavopulmonary (Glenn) circulation (SCPC). By means of MSM with CFD, Schiavazzi and coworkers ${ }^{1}$ studied how varying degrees of stenosis affected the pulmonary flow split ratio and the mean LPA pressure drop. They arrived at the conclusion that the effects of stenosis less than $65 \%$ diameter $(d)$ reduction were minor and thus may not justify the addition of LPA arterioplasty to the SCPC operation.

In steady flow of an ideal fluid (where viscosity is neglected) through a tube, lumen narrowing (reduction in d) will produce a pressure drop, but flow rate will be preserved. In this case, the pressure drop is proportional to $d^{-2}$. In 1846, Poiseuille ${ }^{2}$ used a benchtop apparatus (Figure 1) to show that the pressure drop in a long tube was actually proportional to $d^{-4}$. Although he did not know this, the proportionality constant in his relationship would later be dubbed the viscosity of the fluid.

It gets more complicated than that. The actual pressure drop is not simply predicted by the lumen diameter but also depends on the conditions at the inflow and outflow of the tube-the so-called boundary conditions. For example, in Poiseuille's models, if the inflow pressure were to be held constant and the narrowing increased,

\footnotetext{
From the Division of Cardiothoracic Surgery, The Heart Center at Arnold Palmer Hospital for Children, Orlando, Fla; and the College of Medicine, University of Central Florida, Orlando, Fla.

Disclosures: Author has nothing to disclose with regard to commercial support.

Received for publication Sept 15, 2014; accepted for publication Sept 15, 2014; available ahead of print Oct 8, 2014.

Address for reprints: William M. DeCampli, MD, PhD, Division of Cardiothoracic

Surgery, The Heart Center at Arnold Palmer Hospital for Children, 92 W Miller

St, Orlando, FL 32806 (E-mail: William.decampli@orlandohealth.com Or

William.decampli@ucf.edu).

J Thorac Cardiovasc Surg 2015;149:697-8

$0022-5223 / \$ 36.00$

Copyright (C) 2015 by The American Association for Thoracic Surgery

http://dx.doi.org/10.1016/j.jtcvs.2014.09.024
}

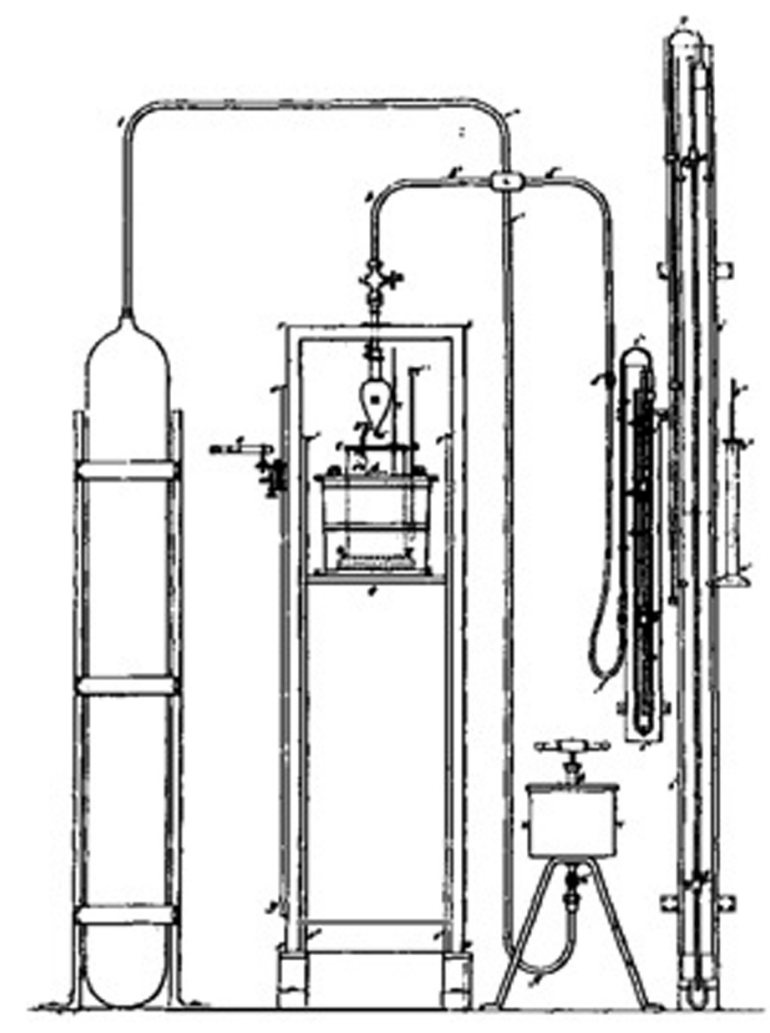

FIGURE 1. A diagram of the benchtop apparatus used by Poiseuille ${ }^{2}$ in 1846 to demonstrate the property of liquid flow now known as viscosity.

then the flow would decrease, and the pressure drop would not be as great as in the case where the inflow flow rate was held constant and the inflow pressure allowed to increase. In the human circulation, these boundary conditions depend on the response of the heart and all other parts of the peripheral vasculature. Here is where MSM with CFD comes into play. Only a detailed, internally consistent, and complete model of the entire circulation can predict the flow split and pressure drop across an LPA stenosis in the SCPC. Such a model is what Schiavazzi and coworkers ${ }^{1}$ produced. If only Poiseuille had had a computer! MSM with CFD is not simulation or game playing. It is calculation that rigorously supplements anatomic and functional imaging in characterizing the normal and abnormal circulation in real patients. Additionally, because one can interact with and modify the model anatomy, cardiac function, and vascular characteristics, CFD facilitates discovery and even surgical planning, all of which are well represented in the current literature.

The current Achilles heel of MSM with CFD is twofold: (1) the accuracy and completeness of the models used for all 
of those other "compartments" (heart, peripheral beds) used in the calculations and (2) the ability to account for changes in the circulation as a result of growth, healing, or progression of disease. For these reasons, CFD investigators must remain very close to clinicians to ensure that they are being realistic and, in particular, not overstating their conclusions. In the current article, Schiavazzi and coworkers ${ }^{1}$ appear to have discovered that clinicians may be overtreating LPA stenosis if the diameter narrowing is less than $65 \%$. In the clinical realm, however, the perceived need for perioperative LPA intervention is based on the level of concern that the SCPC will result in either excessive superior caval pressure or low arterial oxygen saturation. All clinicians see these problems after SCPC from time to time, and in many cases they ameliorate the problem by intervening on either the right pulmonary artery or the LPA. It is curious that none of the current models of Schiavazzi and coworkers ${ }^{1}$ demonstrated any significant decrease in oxygen saturation, even with imposing severe degrees of LPA stenosis. Why is that? It could be because Schiavazzi and coworkers ${ }^{1}$ did not account for systemic venovenous collaterals, which can "open up" when superior caval pressure is just mildly elevated. In addition, the models did not account for imperfect pulmonary function, in which case recruitment of more blood flow to the "better lung" could make a difference. When these factors are not considered in a patient-specific model, CFD may actually suggest an inappropriate clinical decision. All this is to say that MSM with CFD, although a useful supplement to characterize the circulation and tool to investigate certain aspects of cardiovascular physiology, has not reached the point of being sufficiently predictive to be used to make patient-specific clinical decisions. As with any new diagnostic or therapeutic measure, it must ultimately be subjected to appropriate trials before it can be used in patient management. Nevertheless, the future role of CFD in understanding and managing cardiovascular disease continues to be promising.

\footnotetext{
References

1. Schiavazzi DE, Kung EO, Marsden AL, Baker C, Pennati G, Hsi T-Y. Hemodynamic effects of left pulmonary artery stenosis after superior cavopulmonary connection: a patient-specific multiscale modeling study. J Thorac Cardiovasc Surg. 2015;149:689-96.

2. Poiseuille JLM. Recherches expérimentales sur le mouvement des liquides dans les tubes de très-petits diamètres [in French]. Mémoires préséntes par divers savants à l'Académie Royale des Sciences de l'Institut de France. 1846;IX:433-544.
}

Readers who found these articles interesting may also like to read the following papers found in recent and future issues of our sister publications, Seminars in Thoracic and Cardiovascular Surgery and Operative Techniques in Thoracic and Cardiovascular Surgery!

Current Readings: Jeffrey Jacobs. Long Term Management of Patients Undergoing Successful Pediatric Cardiac Surgery. Semin Thorac Cardiovasc Surg. Summer 2014;26(2):132-144.

Current Readings: Brian Kogon. Pulmonary Valve Replacement for Pulmonary Valve Insufficiency in Formerly Repaired Tetralogy of Fallot Patients. Semin Thorac Cardiovasc Surg. Expected publication August 2015.

News and Views: Michael Mitchell. Airway Issues in Infants and Other Children with Congenital Heart Disease. Semin Thorac Cardiovasc Surg. Expected publication August 2015.

Shi-Joon Yoo. MRI as a decision making tool in congenital heart disease surgery. Oper Tech Thorac Cardiovasc Surg. Summer 2014;19(2):152-163.

Pascal Vouhe. Valve Sparing Konno and HOCM in Children. Oper Tech Thorac Cardiovasc Surg. Summer 2014;19(2):164-178. Jose Pedro da Silva. Pulmonary root translocation for anatomical repair of congenitally corrected transposition of the Great Arteries. Oper Tech Thorac Cardiovasc Surg. Expected publication February 2015. 